\title{
A reduced order state space model for aeroelastic analysis in time domain
}

\author{
Clayton R. Marqui ${ }^{\mathrm{a}, *}$, Douglas D. Bueno ${ }^{\mathrm{b}}$, Luiz C.S. Goes ${ }^{\mathrm{a}}$, Paulo J.P. Gonçalves ${ }^{\mathrm{c}}$ \\ ${ }^{a}$ ITA Aeronautical Institute of Technology, Brazil \\ ${ }^{\mathrm{b}}$ UNESP - State University of São Paulo, Fac. Eng. Ilha Solteira, Brazil \\ ${ }^{\mathrm{c}}$ UNESP - State University of São Paulo, Fac. Eng. Bauru, Brazil
}

\section{A R T I C L E I N F O}

\section{Keywords:}

Aeroelasticity

Time-domain representation

Reduced state space model

Laguerre polynomials

\begin{abstract}
A B S T R A C T
The objective of this paper is to describe a new method for modeling aeroelastic system in time domain based on a modification of the Laguerre Polynomials to represent complex quantities. These polynomials are used to approximate the unsteady aerodynamics forces which are defined in the frequency domain using the Doublet Lattice Method (DLM). In this approach, the size of the matrices representing the aeroelastic system remains the same as the matrices representing the structural dynamics behavior. It is an important point since classical state space aeroelastic models include lag states increasing the size of the matrices used to represent the system. The applicability of the method is demonstrated by numerical simulation performed on the benchmark wing structure. The approach offers promise mainly for complex systems such as real aircraft.
\end{abstract}

\section{Introduction}

Fluid structure interaction is probably a field that demands special attention of engineers when designing a new aircraft. Among the problems in this area, the aeroelastic stability analysis (known as flutter analysis) is of special interest. Besides that, the response of structure to gust loads and coupling with control systems has also become an important subject named aeroservoelasticity.

The term aeroservoelasticity defines an area that involves the study of the fluids, structures, servo actuators, control surfaces and design of control units. These are essential equipments in modern fly-by-wire aircraft.

The essential problem in the aeroservoelasticity lies in the fact that most of the methodologies and procedures for fluid-structure analysis are based on the frequency domain. These are well established in the aircraft industry and accepted by certification authorities. For instance, the Doublet Lattice Method (DLM) (Albano and Rodden, 1969) that is often used to represent the unsteady aerodynamic forces is defined in frequency domain.

In terms of the control analysis, most of the modern methods are developed in the time domain using state space representation. Therefore, having a model in the time domain to represent aeroelastic systems is essential for the design of new aircraft. According to Karpel (1981) the importance of writing aerodynamic forces in time domain increased with the progress in active control of aeroelastic systems.

Recent works involving the use of control theory for aeroelasticity stability analysis, include the work of Silva et al. (2006) which an aeroservoelastic plant for HILDA (High Lift-to-Drag Active) wing was identified using experimental data. The impulse response (IR) method was applied in order to identify the state-space model and the generalized predictive control (GPC) method was used to

\footnotetext{
* Corresponding author.

E-mail address: crmarqui@hotmail.com (C.R. Marqui).
} 
develop an AutoRegressive with eXogenous input (ARX) model and the gust load alleviation (GLA) control laws. Haghighat et al. (2012) studied the effect of the control system on the optimal design of the wing and its structure. Both time dependent maneuvers and discret gust excitations are considered in this optimization work. Bueno et al. (2014a) considers the analysis of stability in a range of velocity and altitude, instead of discrete points in the flight envelope. Bueno et al. (2014b) also use the state space representation of aeroelastic system to design a controller for typical section airfoil with nonlinear freeplay. In the work of Bueno et al. (2013), the authors use the concept of Gramian Matrix to study the stability of an airfoil, they claim that the procedure can help reducing the computational time for analysis of many study cases. Bueno et al. (2015) uses the linear matrix inequality theory to study the stability of aeroelastic system containing structural uncertainties.

In classical theory, rational approximations of the unsteady aerodynamic and gust loads have been used to construct state space models. According to the literature, Jones was the first to introduce the use of rational Laplace functions to approximate unsteady loads (Jones, 1940). After this, different formulations have been used to approximate generalized aerodynamic forces for arbitrary motion, as for instance, the approach based on the Chebyshev's polynomials introduced by Botez and colleagues with focus on aeroservoelasticity (Dinu et al., 2005). The most common methods discussed in literature are the Minimum-State Method and the Least Squares Method. The first was proposed by Karpel and involves a nonlinear formulation (Karpel, 1981). The second (called Roger's method) uses common denominator roots to include aerodynamic lag states (Roger, 1977). However, these methods have the drawback of increasing the size of the state space model matrices and this present work introduces an approach to address this problem.

Reduced order models have been explored in different fields of engineering. Particularly for aeroelasticity this term commonly indicates a broad range of models (ROMs, Reduced Order Models) developed to reduce the size of a matrix in CFD (Computational fluid dynamics) solutions. However, herein the reduced model refers to the size of the state space matrices for a linear aeroelastic system considering unsteady aerodynamics. Karpel (1982) presented a new minimum-state method for application on active fluttersuppression and gust-alleviation control systems. The method was based on rational approximation of the unsteady aerodynamic load and demonstrated to result in a considerably lower order state-space model relative to other methods of a comparable accuracy. In Karpel (1990) a reduced-order, time-domain aeroservoelastic modeling was formulated with three types of high-frequency modeelimination techniques: mode truncation, static residualization, and the new dynamic residualization. The formulation was also based on state-space equations of motion where the unsteady aerodynamic force coefficients are represented by a minimum-state rational approximation function. Dowell et al. (1999) provide an overview about reduced order models for usteady aerodynamics, and also, presented a discussion between the following methods: eigenmodes truncation, proper orthogonal decomposition (POD), balanced modes and input/output (transfer function) methods. In Kim et al. (2004), the frequency-domain Karhunen-Loeve method was applied for reduction of the aeroelastic model based on Roger's state-space rational function approximation (RFA). Silva and Bartels (2004) presented a reduced order model for aeroelastic analysis using the modal impulse responses technique and the parallelized CFL3D version 6.0 CFD code. In Munteanu et al. (2005), the authors presented a reduced-order-model based on Volterra identification for non-linear aeroelastic analysis using fluid-structure simulation. Lieu and Farhat (2007) presented a new reduced-order model adaptation scheme based on orthogonal decomposition subspaces and evaluated the aeroelastic models for changes in the free-stream Mach number and angle of attack. Thomas et al. (2007) described a nonlinear reduced-order-modeling technique, based on a Taylor series expansion, for computational aerodynamics and aeroelasticity. Hesse and Palacios (2007) discussed a model reduction approach using the balanced truncation method on the coupled flight dynamics and aeroelastic response of maneuvering flexible aircraft.

In this context, this work presents a different method to obtain the aeroelastic state-space model in the time domain. Laguerre polynomials are used to interpolate the aerodynamic matrices from reduced frequency to Laplace domain. The advantage of the resulting model over traditional rational polynomial curve-fit methods is that there is no introduction of lag states and consequently, the size of the matrices remain the same of the matrices representing the structural dynamics (structural mass, stiffness and damping matrices).

\section{Mathematical model - roger approximation}

Aeroelastic models are characterized by structural matrices (mass, stiffness and damping) obtained by the finite element method and also matrices defining the aerodynamic forces that can be obtained using, for instance, the Doublet Lattice Method. In this paper, the equation of motion of the aeroelastic system under analysis is written as

$$
\mathbf{M} \ddot{\mathbf{u}}(t)+\mathbf{D} \dot{\mathbf{u}}(t)+\mathbf{K u}(t)=q \mathbf{Q}(k) \mathbf{u}(t)
$$

where $\mathbf{M}, \mathbf{K}$ and $\mathbf{D}$ are structural matrices defining the mass, stiffness and damping of the system, respectively. $\mathbf{Q}$ is the aerodynamic matrix which depends on the reduced frequency $k$. u is the generalized displacement vector and $q$ is the dynamic pressure. The solution of this differential equation is obtained assuming harmonic motion, which allows to write the system in Eq. (1) using modal coordinates.

$$
\mathbf{M}_{m} \ddot{\mathbf{u}}_{m}(t)+\mathbf{D}_{m} \dot{\mathbf{u}}_{m}(t)+\mathbf{K}_{m} \mathbf{u}_{m}(t)=q \mathbf{Q}_{m} \mathbf{u}_{m}(t)
$$

The aerodynamic matrix $\mathbf{Q}$ is defined in the frequency domain and it can not be transformed to time domain directly, therefore approximations are often used.

An example is the rational function approximation proposed by Roger (1977). Roger's approximation is defined in the frequency (Laplace) domain containing a polynomial term representing the forces acting on the airfoil directly related to the displacements $\mathbf{u}(t)$ 
and it's first and second derivatives. It has also a rational term representing the influence of the wake acting on the airfoil with a time delay. This function is shown in Eq. (3) and it is used as a reference for comparison with the method proposed in this paper.

$$
\mathbf{Q}_{m}(s)=\left[\mathbf{Q}_{m 0}+\mathbf{Q}_{m 1} s\left(\frac{b}{V}\right)+\mathbf{Q}_{m 2} s^{2}\left(\frac{b}{V}\right)^{2}+\sum_{j=1}^{n_{\text {lag }}} \mathbf{Q}_{m(j+2)}\left(\frac{s}{s+\frac{b}{V} \beta_{j}}\right)\right] \mathbf{u}_{m}(s)
$$

where $s$ is the Laplace variable, $n_{\text {lag }}$ is the number of lag terms and $\beta_{j}$ is the $j$ th lag parameter $\left(j=1, \ldots, n_{\text {lag }}\right)$. The subscript $m$ represents the modal coordinates.

Substituting Eq. (3) into Eq. (2) and after some rearrangements, the classical aeroelastic equation of motion in a state space representation is obtained

$$
\dot{\mathbf{x}}(t)=\mathbf{A x}(t) \quad \text { and } \quad \mathbf{y}(t)=\mathbf{C x}(t)
$$

where $\mathbf{x}(t)=\left\{\dot{\mathbf{u}}_{m} \mathbf{u}_{m} \mathbf{u}_{a m}\right\}^{T}$ is the state vector and $\mathbf{u}_{a m}$ is a vector containing lag states. The matrix of outputs $\mathbf{C}=\left[\mathbf{C}_{v} \mathbf{C}_{d}\right]$ has dimension $2 m \times m\left(2+n_{\text {lag }}\right)$, where $\mathbf{C}_{v}$ and $\mathbf{C}_{d}$ are the velocity and displacement output matrices, respectively. The matrix $\mathbf{A}$ is presented in the following form (Wright and Cooper, 2007):

$$
\mathbf{A}=\left[\begin{array}{ccccc}
\mathbf{M}_{a m}^{-1} \mathbf{D}_{a m} & \mathbf{M}_{a m}^{-1} \mathbf{K}_{a m} & -q \mathbf{M}_{a m}^{-1} \mathbf{Q}_{m 3} & \cdots & \mathbf{M}_{a m}^{-1} \mathbf{Q}_{m\left(2+n_{l a g}\right)} \\
\mathbf{I} & \mathbf{0} & \mathbf{0} & \cdots & \mathbf{0} \\
\mathbf{I} & \mathbf{0} & \left(-\frac{V}{b}\right) \beta_{1} \mathbf{I} & \mathbf{0} & \cdots \\
\vdots & \vdots & \mathbf{0} & \ddots & \cdots \\
\mathbf{I} & \mathbf{0} & \vdots & \cdots & \left(-\frac{V}{b}\right) \beta_{n_{l a g}} \mathbf{I}
\end{array}\right]
$$

where

$$
\mathbf{M}_{a m}=\mathbf{M}_{m}-q\left(\frac{b}{V}\right)^{2} \mathbf{Q}_{m 0}, \quad \mathbf{D}_{a m}=\mathbf{D}_{m}-q\left(\frac{b}{V}\right) \mathbf{Q}_{m 1}, \quad \mathbf{K}_{a m}=\mathbf{K}_{m}-q \mathbf{Q}_{m 2}
$$

Note that the size of the state Matrix A is $m\left(2+n_{l a g}\right) \times m\left(2+n_{l a g}\right)$, i.e., it is much larger than twice the size of the structural matrices due to lag states inclusions, where $m$ is number of degrees of freedom for physical coordinates or the number of modes for generalized coordinates.

\section{Reduced order state space aeroelastic model}

An alternative representation of the aeroelastic system obtained using Roger's approximation is proposed using Laguerre Polynomials. This representation does not require the use of lag states, but it can cause an poor estimation of the damping in the system. In this sense, a procedure to improve the damping ratio estimation is presented in the following sections.

\subsection{Laguerre polynomials}

According to Iyanaga and Kawada (1980) and Zwillinger (1997), the Laguerre differential equation is defined as

$$
k \frac{d^{2} f}{d k^{2}}+(\lambda+1-k) \frac{d f}{d k}+n f=0
$$

where $\lambda, n$ and $k \in \mathbb{R}$. The Laguerre polynomials are solutions to the Laguerre differential equation (with $\lambda=0$ ), which is defined as

$$
k \frac{d^{2} f}{d k^{2}}+(1-k) \frac{d f}{d k}+n f=0
$$

where $n$ is a constant and $k \in \mathbb{R}$. Using the Frobenius method, it is possible to substitute a series solution about the regular singular point $k=0$ as

$$
f(k, x)=\sum_{r=0}^{\infty} a_{r} k^{x+r}
$$

and obtain a two-term recursion relation

$$
a_{r+1}=a_{r} \frac{(x+r-n)}{(x+r+1)^{2}}
$$

In this case, the indicial equation has a double root, $x=0$, where the two linearly independent solutions are give as

$$
f(k, 0) \text { and }\left.\frac{\partial f(k, x)}{\partial x}\right|_{x=0}
$$

The second solution diverges logarithmically as $k \rightarrow 0$. Hence for finite values, only the first solution is considered, $f(k, 0)$, which 
has the recursion relation

$$
a_{r+2}=-a_{r} \frac{(n-r)}{(n+1)^{2}}
$$

providing the infinite series

$$
f(k)=a_{0}\left[1-\frac{n k}{1^{2}}+\frac{n(n-1)}{(2)^{2}} k^{2}+\ldots+\frac{(-1)^{r} n(n-1) \ldots(n-r+1)}{(r)^{2}} k^{r}+\ldots\right]
$$

From the recursion relation shown in Eq. (12), in the limit of $r \rightarrow \infty$, the ratio of two successive terms has the limit $a_{r+1} / a_{r} \rightarrow 1 / r$ and the series diverges for large values of $k$. If $n$ is restricted to integer values, it is possible to obtain finite polynomial solutions as

$$
f(k)=a_{0} \sum_{r=0}^{n}(-1)^{r} \frac{n(n-1) \ldots\left(n-r_{1}\right)}{(r !)^{2}} k^{r} \quad \text { or, } \quad f(k)=a_{0} \sum_{r=0}^{n}(-1)^{r} \frac{n ! k^{r}}{(n-r) !(r !)^{2}}
$$

The Laguerre polynomials are defined by setting $a_{0}=1$ in Eq. (14) as

$$
P_{n}^{(L a g)}(k)=\sum_{r=0}^{n}(-1)^{r} \frac{n ! k^{r}}{(n-r) !(r !)^{2}}
$$

There are other definitions of Laguerre polynomials as, for an instance, the Rodriguez formula which involves a derivative term. However, Eq. (15) has a convenient format to write aerodynamic forces and loads because $k$ appears explicitly.

\subsection{Complex laguerre polynomials}

Laguerre polynomials are originally written using real variables. Consider, for instance, the polynomial expansion written as

$$
L_{z}\left(s^{\prime}\right)=\alpha_{0}\left(s^{\prime}\right)^{0}+\alpha_{1}\left(s^{\prime}\right)^{1}+\cdots+\alpha_{x}\left(s^{\prime}\right)^{x}+\cdots+\alpha_{z}\left(s^{\prime}\right)^{z},
$$

or, for simplicity,

$$
L_{z}\left(s^{\prime}\right)=\sum_{x=0}^{z} \alpha_{x}\left(s^{\prime}\right)^{x}
$$

Laguerre polynomials are originally defined for real variables. In this work, a modification is proposed to represent complex quantities, writing the complex variable $s^{\prime}=j k=j \omega(b / V)=(b / V) s$, where $s$ is the Laplace variable and $k$ is the reduced frequency. reminding that

$$
\alpha_{x}=(-1)^{x} \frac{z !}{(z-x) !(x !)^{2}}
$$

does not dependent on $k$ and the modified Laguerre polynomials are defined in complex domain, i.e., $L_{z} \in C$.

For high values of $x$, the reduce frequency simplifies to $k=\omega(b / V)$, and the term $\left(s^{\prime}\right)^{x}$ can generate sparse matrices. Thus, $\widetilde{L}_{z}$ is defined, such that

$$
\tilde{L}_{z}\left(s^{\prime}\right)=\sum_{x=0}^{z} \alpha_{x}\left(s^{\prime}\right)^{\tilde{x}}, \quad \text { where } \quad \tilde{x}= \begin{cases}x, & \text { if } x \leq 2 \\ 0, & \text { if } x>2 \text { and odd } \\ 1, & \text { if } x>2 \text { and even }\end{cases}
$$

Assuming that each element in the matrix of aerodynamic influence coefficients $\mathbf{Q}(k)$ can be written for the $k$-th reduced frequency as a linear combination of the complex Laguerre polynomials, then

$$
q_{i j}(k)=\sum_{z=0}^{r} C_{z}^{(i j)} \tilde{L}_{z}
$$

where the coefficients $C_{z} \in \mathcal{R}$ and $\widetilde{L}_{z} \in C$, such that $q_{i j}(k)=q_{i j}^{R}(k)+j q_{i j}^{I}(k)$. After rearrangements (see Appendix A), it is possible to demonstrate that aerodynamic forces shown in the right-hand side of Eq. (1) can be written as

$$
\mathbf{F}_{a}(t)=q\left\{\sum_{x} \widetilde{\mathbf{Q}}^{(x)} \mathbf{u}(t)+\left(\frac{b}{V}\right) \sum_{y} \widetilde{\mathbf{Q}}^{(y)} \dot{\mathbf{u}}(t)+\left(\frac{b}{V}\right)^{2} \widetilde{\mathbf{Q}}^{(2)} \dot{\mathbf{u}}(t)\right\}
$$

Thus, the aeroelastic model in the time domain is rewritten as

$$
\left[\mathbf{M}-q\left(\frac{b}{V}\right)^{2} \widetilde{\mathbf{Q}}^{(2)}\right] \ddot{\mathbf{u}}(t)=\left[-\mathbf{D}+q\left(\frac{b}{V}\right) \sum_{y} \widetilde{\mathbf{Q}}^{(y)}\right] \dot{\mathbf{u}}(t)+\left[-\mathbf{K}+q \sum_{x} \widetilde{\mathbf{Q}}^{(x)}\right] \mathbf{u}(t)
$$

and the new state space aeroelastic system is defined for the state vectors $\mathbf{x}=\left\{\begin{array}{ll}\dot{\mathbf{u}} & \mathbf{u}\end{array}\right\}^{T}$ and 


$$
\mathbf{A}=\left[\begin{array}{cc}
\mathbf{M}_{a}^{-1} \mathbf{D}_{a} & \mathbf{M}_{a}^{-1} \mathbf{K}_{a} \\
\mathbf{I} & \mathbf{0}
\end{array}\right]
$$

where

$$
\mathbf{M}_{a}=\left[\mathbf{M}-q\left(\frac{b}{V}\right)^{2} \widetilde{\mathbf{Q}}^{(2)}\right], \quad \mathbf{D}_{a}=\left[-\mathbf{D}+q\left(\frac{b}{V}\right) \sum_{y} \widetilde{\mathbf{Q}}^{(y)}\right], \quad \mathbf{K}_{a}=\left[-\mathbf{K}+q \sum_{x} \widetilde{\mathbf{Q}}^{(x)}\right]
$$

Note that matrix A has dimension $2 \mathrm{~m} \times 2 \mathrm{~m}$ (the same order of structural model). Similarly to what was described in Section 2, this model also can be written using modal coordinates. In this case, the procedure to obtain the state space dynamic matrix (Eq. (23)) is the same.

\subsection{Correction of the damping matrix}

The introduced approach to obtain the state space representation can result in aeroelastic damping ratios with some differences in comparison with those values obtained in frequency domain by the $p k$ method. In practice, this differences are generated by numerical truncation during the proposed solution. Once the values of frequencies, in general, are largest than damping ratios ones, only these last one must be corrected. In this sense, this poor estimation can be overcome by adjusting scaling the damping using a weight matrix. The details for this procedure are explained hereafter.

To apply a correction to the aeroelastic damping matrix $\left(\mathbf{D}_{a}\right)$ is previously solved the eigenvalue problem for the undamped aeroelastic system, such that

$$
\left[\mathbf{K}_{a}-\left(\omega_{j}^{(\text {und })}\right)^{2} \mathbf{M}_{a}\right] \varphi_{j}=0
$$

where $\omega_{j}^{(\text {und })}$ is the $\mathrm{j}$-th frequency of the undamped aeroelastic system and $\varphi_{j}$ is the $\mathrm{j}$-th eigenvector. Note that $\varphi_{j} \in \mathbb{R}$, therefore, these are not the aeroelastic eigenvectors.

The corrected aeroelastic damping matrix is obtained assuming viscous damping coefficients for each eigenfrequency obtained from the solution of problem in Eq. (25)

$$
\mathbf{D}_{a}^{(v i s c)}(j, j)=-2 \xi_{j}^{a} \omega_{j}^{(\text {und })}
$$

where $\xi_{j}^{a}$ is the $\mathrm{j}$-th damping ratio of the original aeroelastic system, obtained through the solution of the p-k method (in the frequency domain). Note that $\mathbf{D}_{a}^{(v i s c)}(i, j)=0$ for $i \neq j$, i. e., $\mathbf{D}_{a}^{(\text {visc })}$ is a diagonal matrix, that can be rewritten

$$
\mathbf{D}_{a}^{(\text {visc })}=-2 \boldsymbol{\Xi}^{a} \mathbf{\Omega}^{(\text {und })}
$$

Finally, the correction is performed using a weight matrix $\mathbf{W}_{\text {damp }}$

$$
\mathbf{D}_{a}=\mathbf{W}_{\text {damp }} \mathbf{D}_{a}^{(\text {visc })}
$$

where $\mathbf{D}_{a}$ is the corrected aeroelastic damping matrix. The corrections factors $\mathbf{W}_{\text {damp }}(i, j)$ are chosen such that the eigenvectors of $\mathbf{A}$ represent the damping factors $\xi_{j}{ }^{a}$ obtained using the $\mathrm{p}-\mathrm{k}$ method.

\section{Numerical simulations}

The proposed method was verified through numerical simulations using the AGARD 445.6 wing. This classical system has been used by different author as showed in references (Pahlavanloo, 2007; Yates, 1988). Also, Haase et al. (2003) show a considerable number of numerical simulations. For this work were considered the same flight conditions showed in Bueno et al. (2014a).

The model was obtained using Finite Element method (Nastran Software), which consists of 200 shell (CQUAD4) elements and 231 nodes as illustrated in Fig. 1.

The wing is modeled with plate elements as a single layer orthotropic material and the model consisted of 231 nodes and 200 elements. The thickness distribution was governed by the airfoil shape. The material properties used shown in Table 1 .

The model has a length of reference $2 b=0.5578 \mathrm{~m}$, a sweep angle is 45 degrees at the quarter chord line, a semi-span of $0.762 \mathrm{~m}$

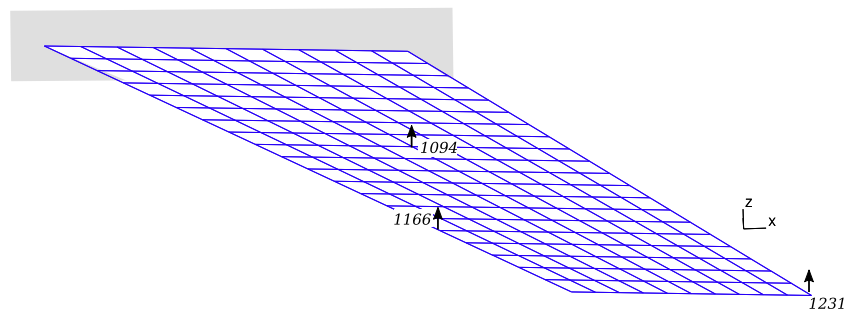

Fig. 1. Finite element mesh of the AGARD wing indicating the nodes 1094, 1166 and 1231 used for comparisons of the proposed methodology. 
Table 1

Material properties of the AGARD 445.6 Wing.

\begin{tabular}{ll}
\hline Property & Value \\
\hline Young Modulus $E_{1}$ & $3.1511 \mathrm{GPa}$ \\
Young Modulus $E_{2}$ & $0.4162 \mathrm{GPa}$ \\
Poisson Coefficient $v$ & 0.31 \\
Shear Modulus $G$ & $0.4392 \mathrm{GPa}$ \\
Density $\rho$ & $321.92 \mathrm{~kg} / \mathrm{m}^{3}$ \\
\hline
\end{tabular}

\section{Table 2}

Reduced frequencies used in the numerical study.

\begin{tabular}{|c|c|c|c|}
\hline 0.001 & 0.002 & 0.005 & 0.010 \\
\hline 0.050 & 0.100 & 0.200 & 0.300, \\
\hline 0.500 & 0.600 & 0.800 & 1.000, \\
\hline 1.500 & 2.000 & 3.000 & 4.000 \\
\hline
\end{tabular}

and a taper ratio of 0.66. More detailed information about AGARD wing can be easily obtained from literature; see Haase et al. (2003), Pahlavanloo (2007) and others.

The Aerodynamic and structural matrices are obtained by NASTRAN program (solution 145) considering Mach number equal to 0.50 and $\rho_{R E F}=1.225 \mathrm{~kg} / \mathrm{m}^{3}$. The values of reduced frequencies are show in Table 2. The main flutter mechanism occur at $115 \mathrm{~m} / \mathrm{s}$ EAS (Equivalent Air Speed) with the coupling of first and second aeroelastic mode shapes (see Bueno et al., 2014a for details).

\subsection{Verification of the proposed methodology}

To verify the proposed methodology, results are compared with Roger's approximation for the aerodynamic influence matrix. For this, the lag parameters adopted to represent the system in time domain were $\beta_{1}=0.55, \beta_{2}=1.40, \beta_{3}=1.90$ and $\beta_{4}=2.90\left(n_{\text {lag }}=4\right)$. These values were adopted such that the poles of the system in time domain were about the same of the poles in frequency domain.

The dimensions of the dynamic matrix A obtained using the Roger's Approximation and the matrix obtained using the proposed method (Laguerre Polynomials) are compared in Fig. 2. This figure was developed considering the first twenty mode shapes ( $m=20)$. The Roger's approximation produces a system with order $\left(m\left(2+n_{\text {lag }}\right)=120\right)$ while the approximation using Laguerre Polynomials produces a matrix with order $2 m=40$.

Numerical simulations where performed with the objective of calculating the aeroelastic frequencies and damping as a function of the airspeed. The airspeed range considered in the analysis was defined from $44 \mathrm{~m} / \mathrm{s}$ to $58 \mathrm{~m} / \mathrm{s}$ of equivalent airspeed (EAS).

The comparisons for frequencies are shown in Fig. 3 while Fig. 4 shows the comparison for damping. It can be seen that the frequencies are almost the same for the two methods. However, it is possible to notice a considerable difference for the estimation of damping.

Because of the differences in the aeroelastic damping results, the correction procedure described in Eq. (28) was applied in the system. A new estimation of damping was calculated and results were compared in Fig. 5 for the specific airspeed (52.3 m/s EAS).

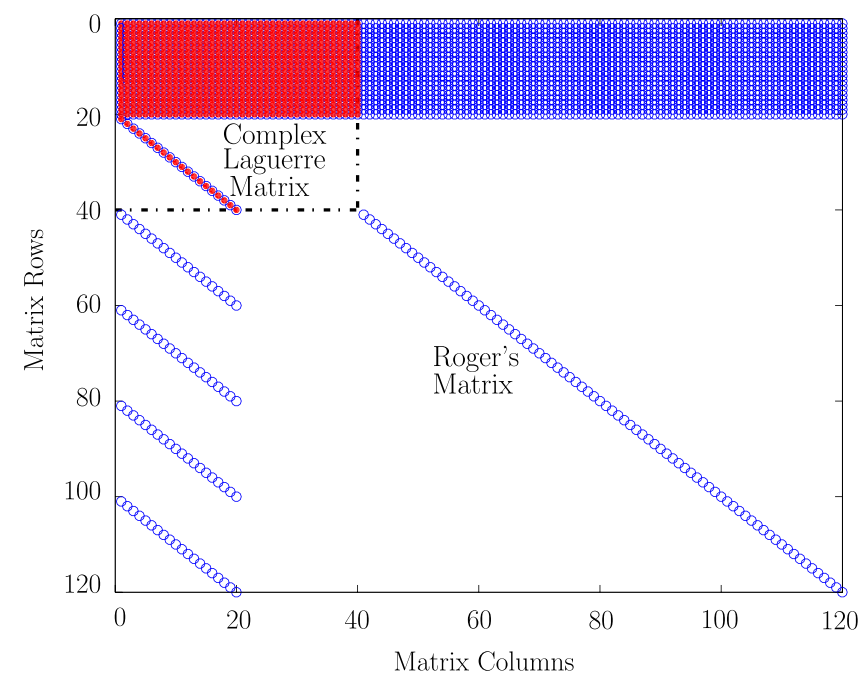

Fig. 2. Comparison of matrices sizes. The red dots illustrate the non-zero elements of the matrix obtained using the laguerre polynomials and the blue circles represent the non-zero elements of the matrix obtained using the Roger's approximation. 
- Doublet Lattice

- Roger

$\square$ Complex Laguerre (uncorrected)

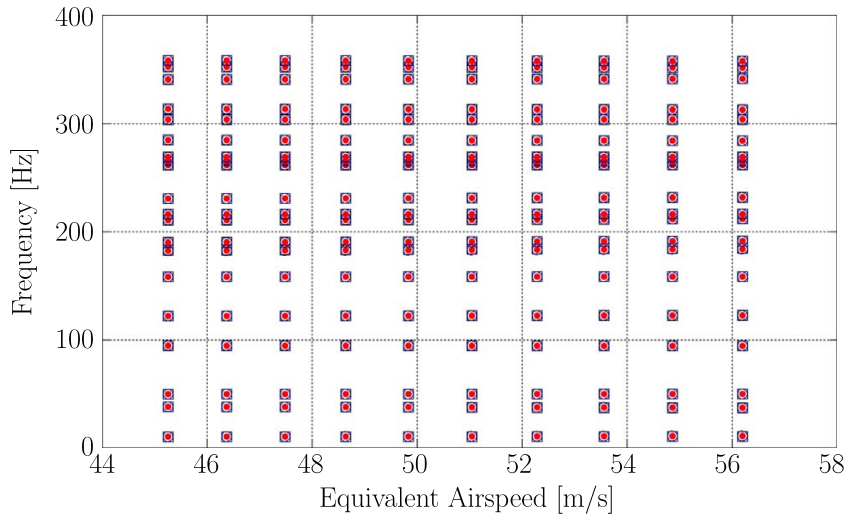

Fig. 3. The aeroelastic frequencies as a function of the airspeed (V-F Diagram).

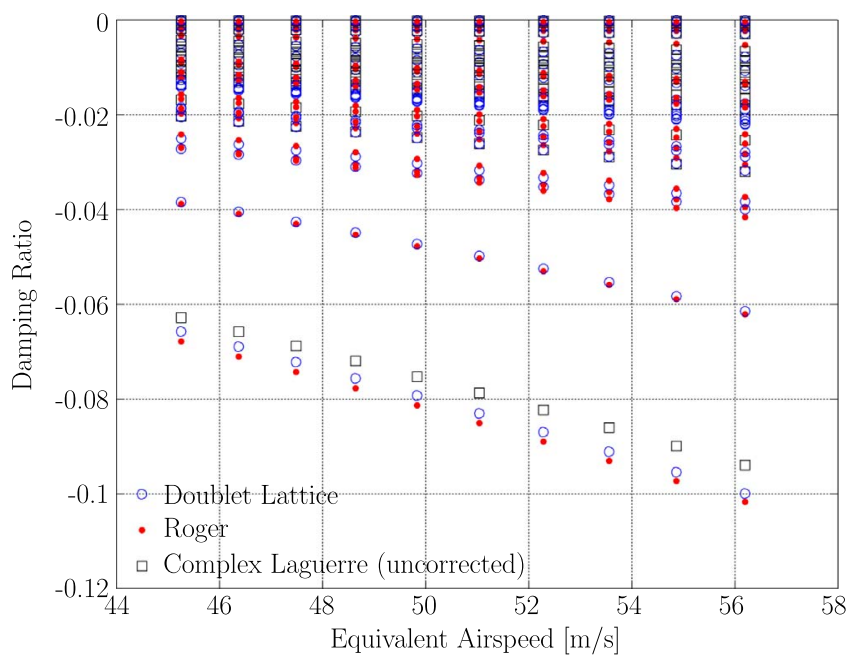

Fig. 4. The aeroelastic damping as a function of the airspeed (V-G Diagram).
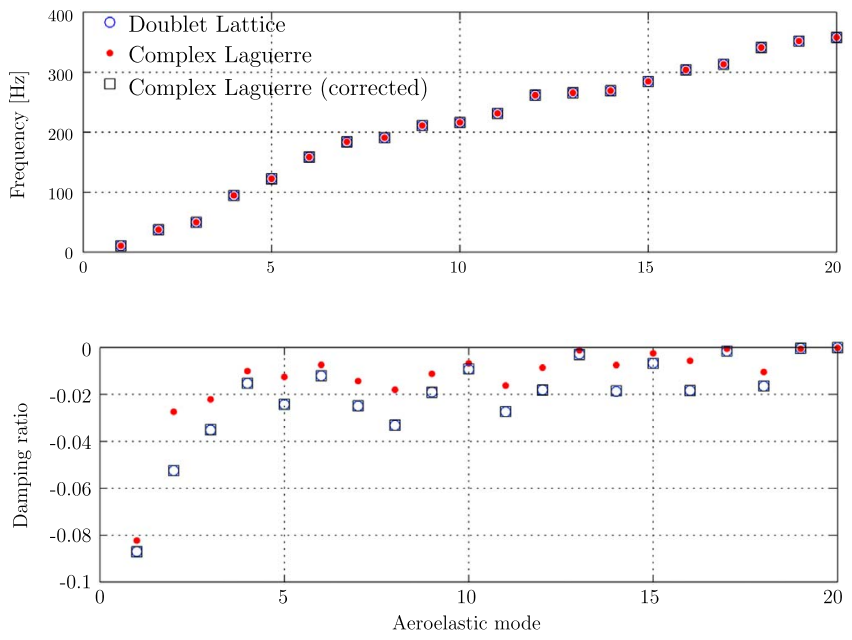

Fig. 5. Frequencies and damping ratios of the aeroelastic system ( $52.3 \mathrm{~m} / \mathrm{s}$ EAS). 

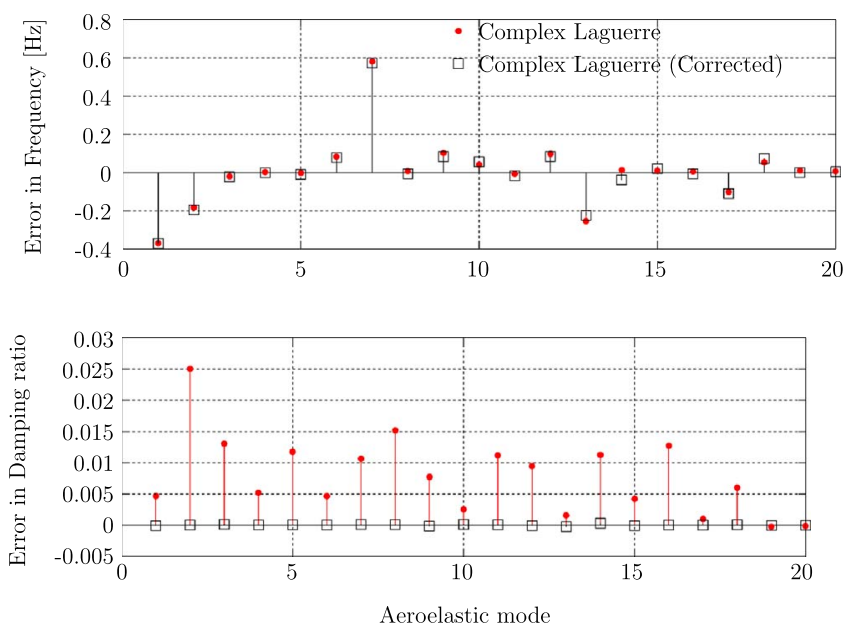

Fig. 6. Absolute errors in the frequencies and damping ratios of the aeroelastic system (52.3 m/s EAS).
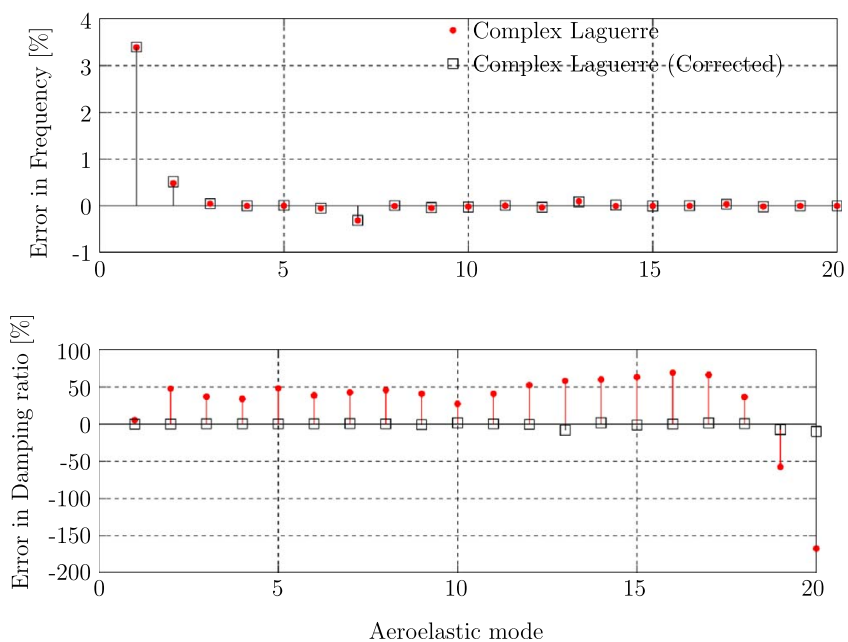

Fig. 7. Relative errors in the frequencies and damping ratios of the aeroelastic system (52.3 m/s EAS).

It is possible to verify that the aeroelastic frequencies remain almost unchanged after the damping correction process. The reason for this is because the procedure changes mainly the matrix related to the damping affecting more the real part of the eigenvalues. Both absolute and relative errors, before and after the damping correction, were calculated and shown in Figs. 6 and 7 .

After applying the correction in the damping matrix, the response to initial conditions were calculated using the individual modal displacements of the first four aeroelastic modes. These results are shown in Figs. 8(a), 9(a), 10(a) and 11(a) (The adopted initial condition was $x_{0}=0.2$ and no initial velocity). Also, a FFT (fast fourier transform) was applied to these responses and the results are shown in Figs. 8(b), 9(b), 10(b) and 11(b), respectively.

Similarly, the free response due to initial conditions in physical coordinates for the nodes 1094, 1166 and 1231 (identified in Fig. 1) were calculated and shown in Figs. 12 up to 14 and the respective Fast Fourier Transform. Many numerical solutions were performed with different initial conditions and all results are very consistent with that case included in the paper.

\section{Final remarks}

This paper has proposed a new methodology to represent aeroelastic systems in time domain to study arbitrary motion. The method is based on the modification of the Laguerre Polynomials with complex variables, allowing to write the aerodynamic forces in the time domain. The advantage of the method is related to the matrix size reduction (when compared to the traditional methods of the literature). A damping correction method was also developed such that the aeroelastic damping are adjusted to the values obtained in the frequency domain. The method was compared with the traditional methodologies by numerical simulation, showing good agreement between the results. 


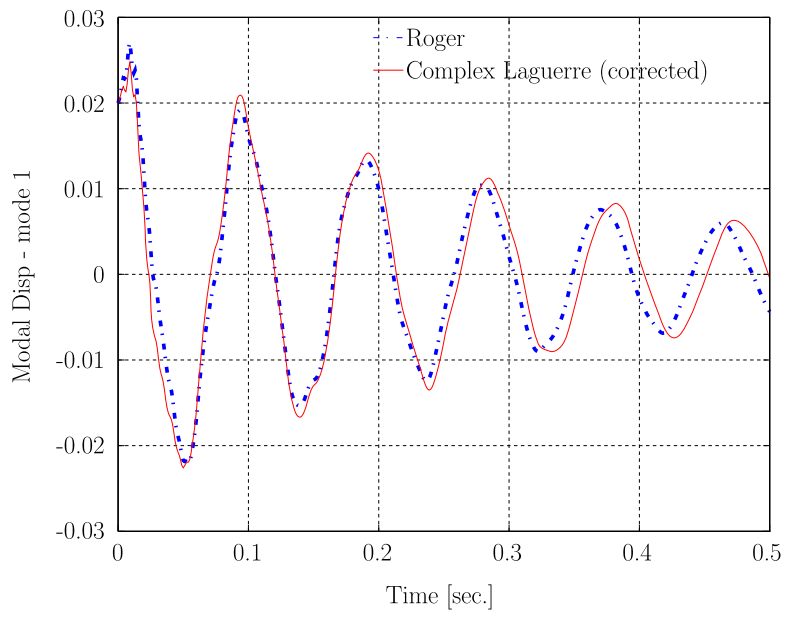

(a)

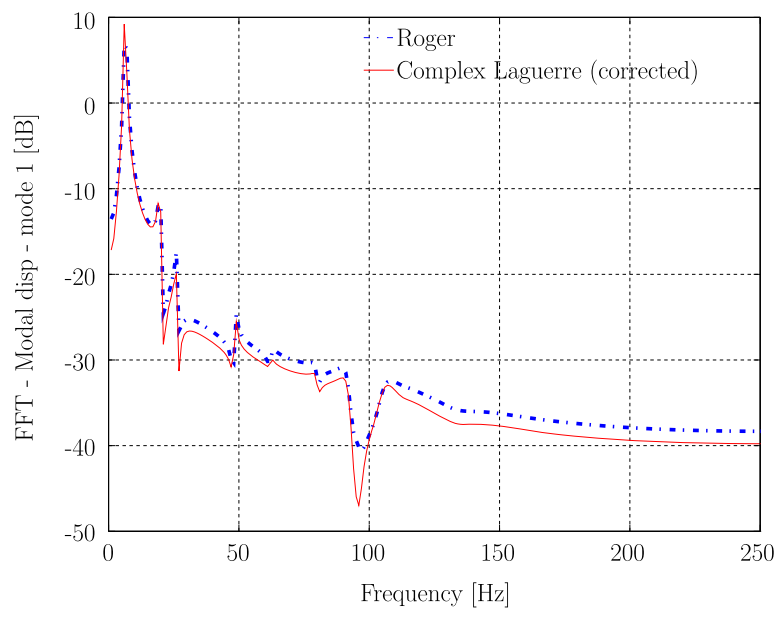

(b)

Fig. 8. Comparison of Modal for the first mode ( $52.3 \mathrm{~m} / \mathrm{s}$ EAS) (a) response to initial condition (b) the respective frequency response.

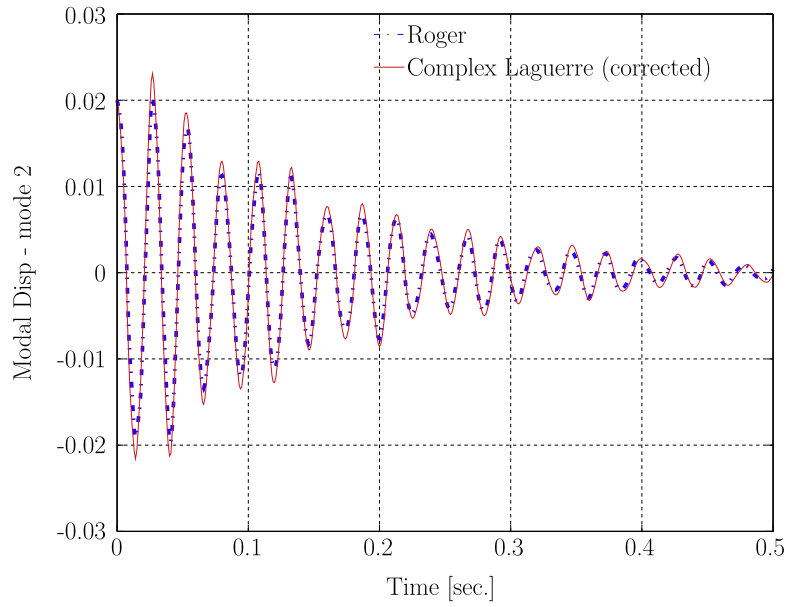

(a)

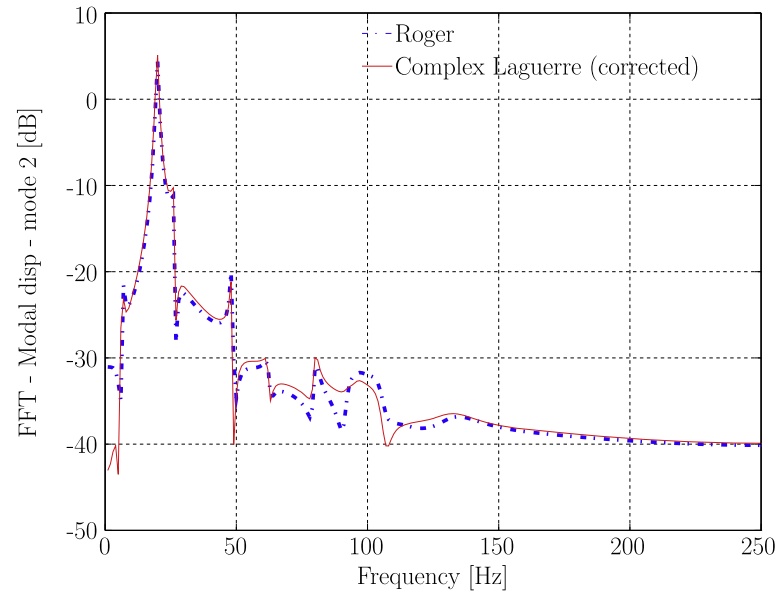

(b)

Fig. 9. Comparison of Modal for the second mode (52.3 m/s EAS) (a) response to initial condition (b) the respective frequency response.

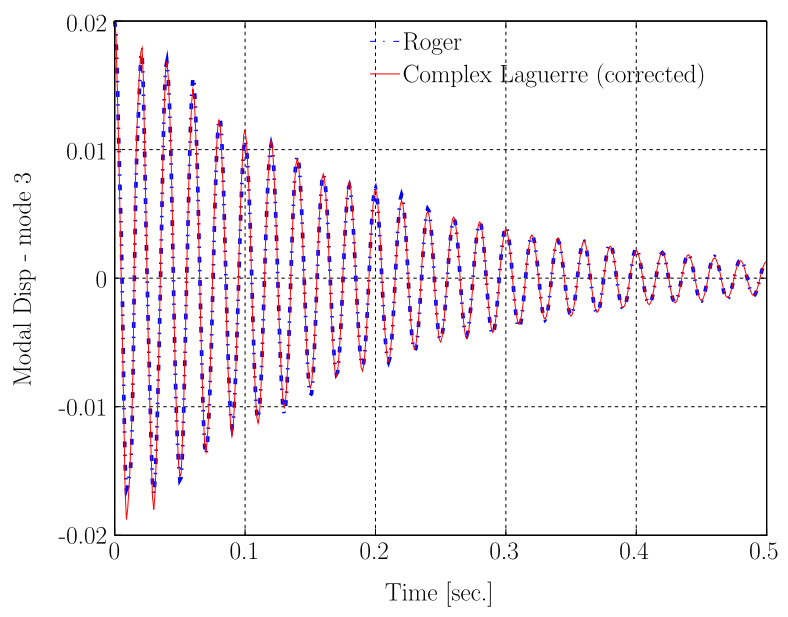

(a)

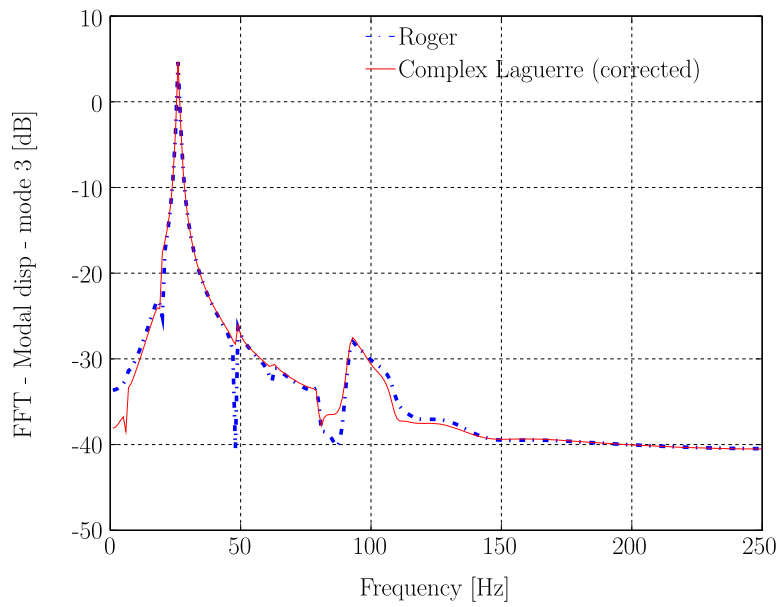

(b)

Fig. 10. Comparison of Modal for the third mode (52.3 m/s EAS) (a) response to initial condition (b) the respective frequency response. 


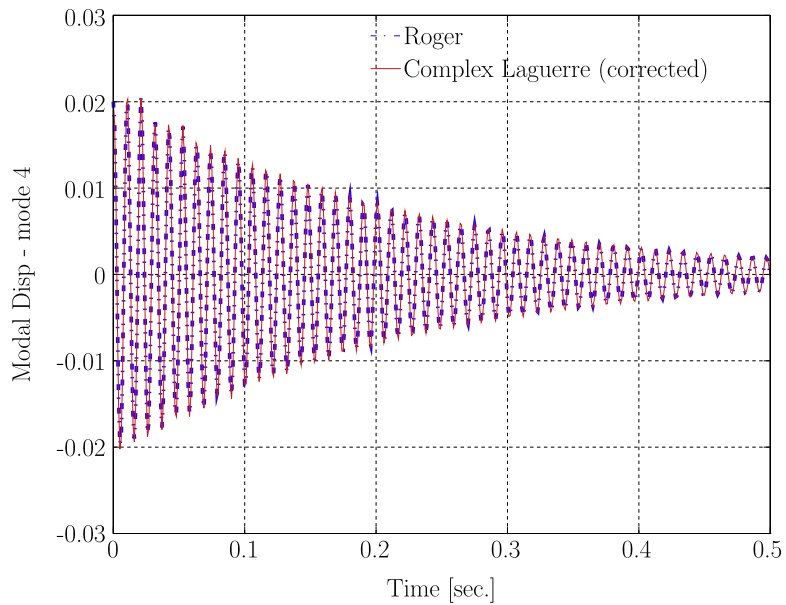

(a)

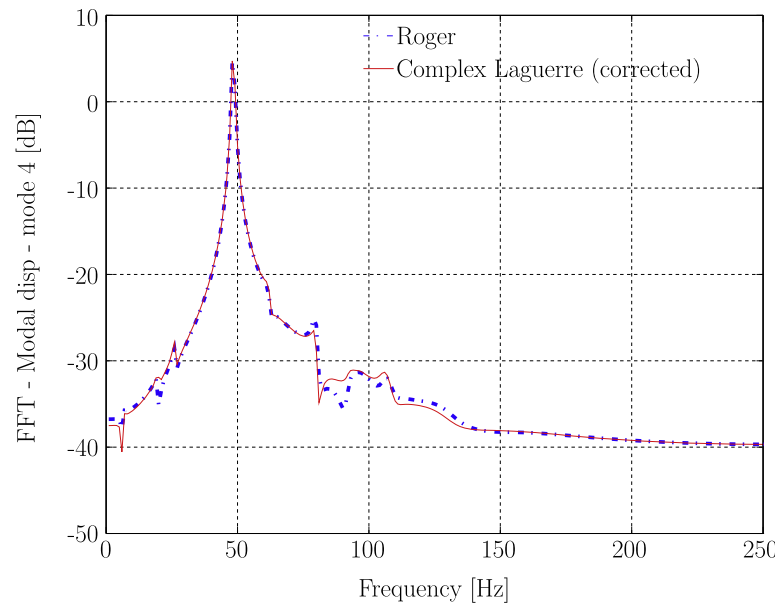

(b)

Fig. 11. Comparison of Modal for the fourth mode (52.3 m/s EAS) (a) response to initial condition (b) the respective frequency response.

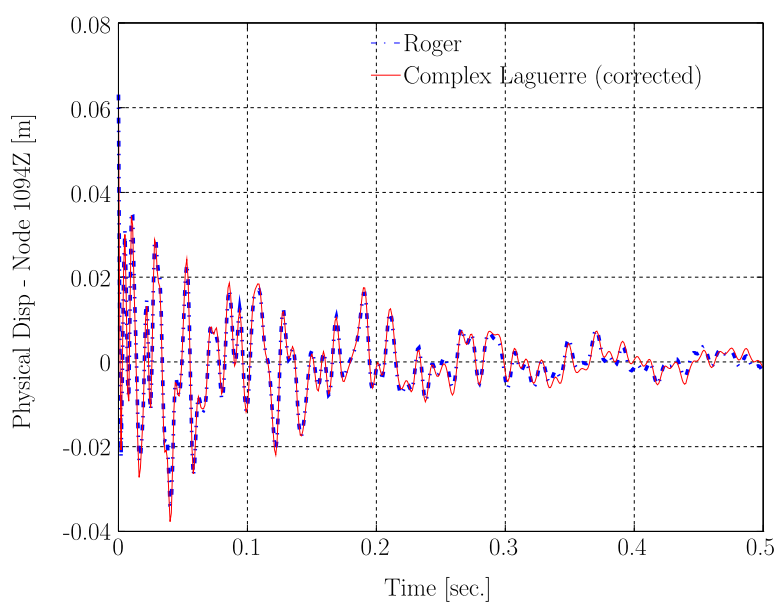

(a)

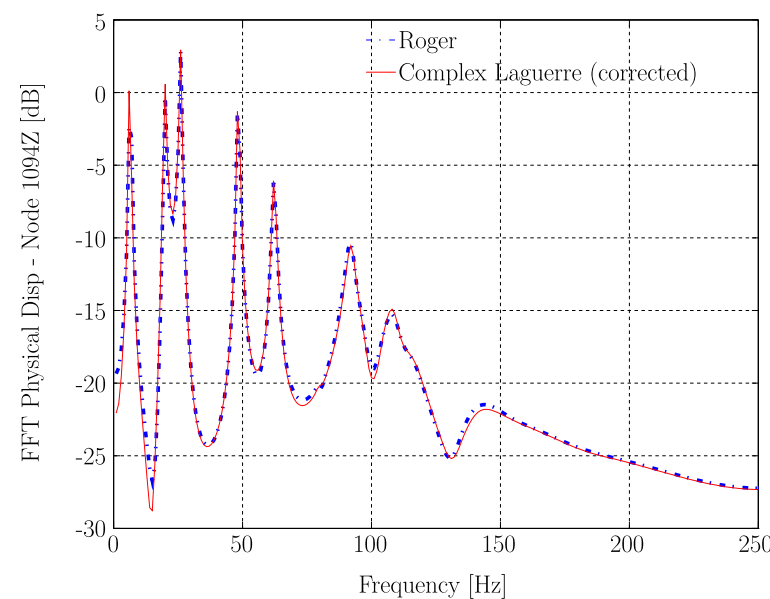

(b)

Fig. 12. Comparison of displacement of the node 1094 (z-direction). (a) Response to initial condition and (b) the frequency response (52.3 m/s EAS).

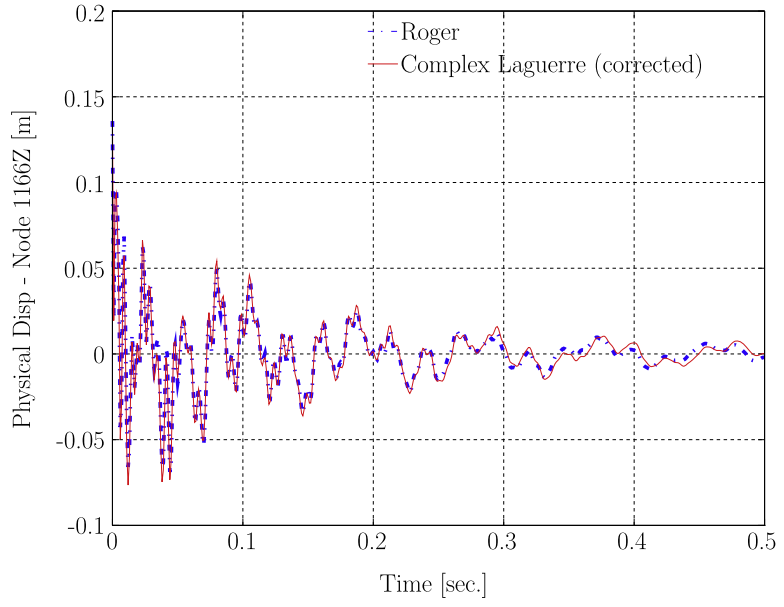

(a)

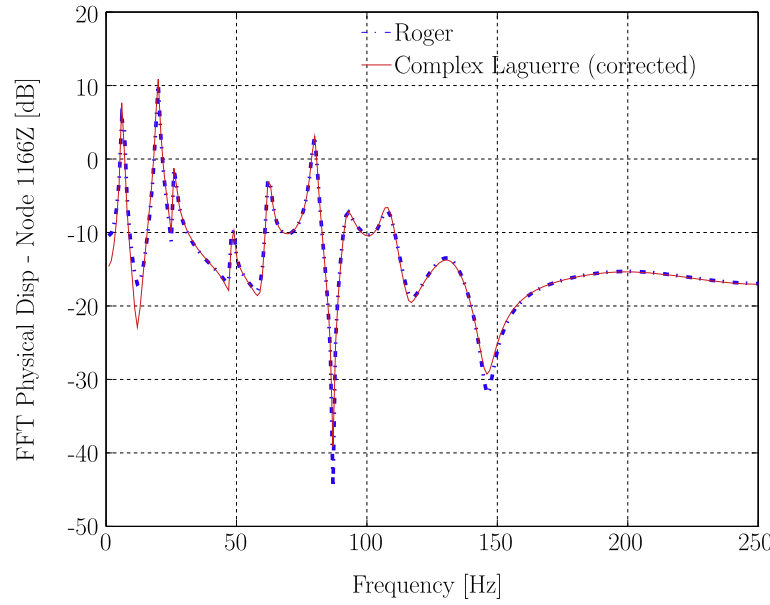

(b)

Fig. 13. Comparison of displacement of the node 1166 (z-direction). (a) Response to initial condition and (b) the frequency response (52.3 m/s EAS). 


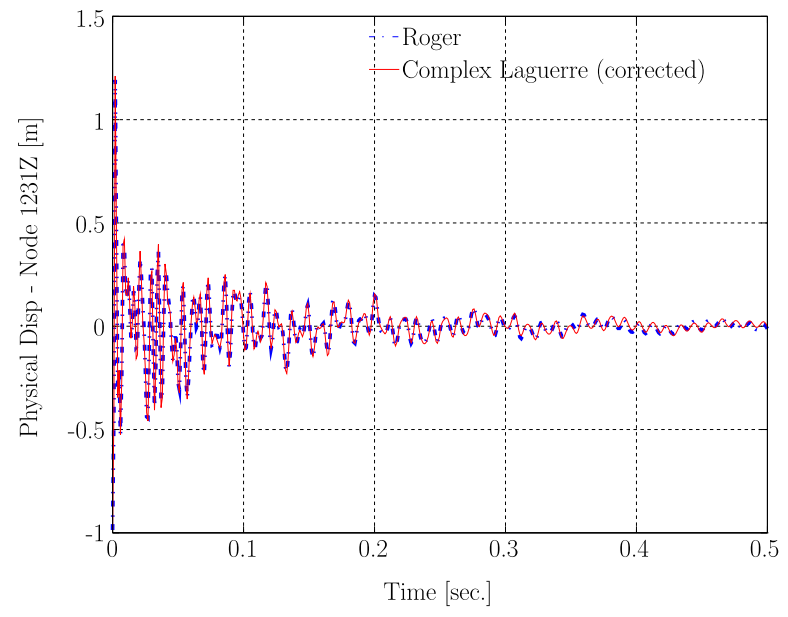

(a)

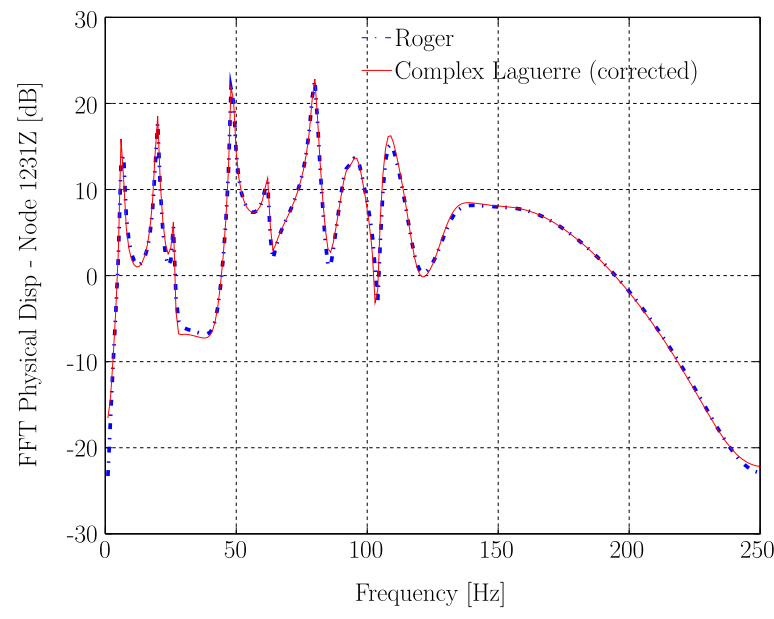

(b)

Fig. 14. Comparison of displacement of the node 1231 (z-direction). (a) Response to initial condition and (b) the frequency response (52.3 m/s EAS).

\section{Appendix A. Complex laguerre polynomials}

This section presents in detail the algebraic manipulation to obtain the aerodynamic forces approximation using a modified form of the Laguerre polynomials. The first step to demonstrate the result presented in Eq. (21) is to rewrite Eq. (20) as

$$
\left[\begin{array}{llll}
C_{0}^{(i j)} & C_{1}^{(i j)} & \ldots & C_{r}^{(i j)}
\end{array}\right]\left[\begin{array}{c}
\widetilde{L}_{0}^{R}+j \widetilde{L}_{0}^{I} \\
\widetilde{L}_{1}^{R}+j \widetilde{L}_{1}^{I} \\
\vdots \\
\widetilde{L}_{r}^{R}+j \widetilde{L}_{r}^{I}
\end{array}\right]=q_{i j}^{R}(k)+j q_{i j}^{I}(k)
$$

or

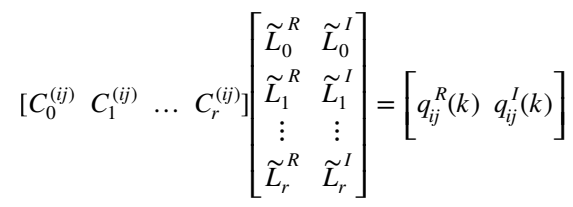

In the matrix form:

$$
\mathbf{C}^{(i j)} \widetilde{\mathbf{L}}(k)=\overline{\mathbf{q}}_{i j}(k)
$$

Eq. (A.3) must be write for any reduced frequency $k$, such that

$$
\begin{gathered}
\mathbf{C}^{(i j)} \widetilde{\mathbf{L}}\left(k_{1}\right)=\overline{\mathbf{q}}_{i j}\left(k_{1}\right) \\
\mathbf{C}^{(i j)} \widetilde{\mathbf{L}}\left(k_{2}\right)=\overline{\mathbf{q}}_{i j}\left(k_{2}\right) \\
\vdots \\
\mathbf{C}^{(i j)} \widetilde{\mathbf{L}}\left(k_{3}\right)=\overline{\mathbf{q}}_{i j}\left(k_{3}\right)
\end{gathered}
$$

In order to obtain the coefficients $\mathbf{C}^{(i j)}$, it is possible to multiply both left and right sides of Eq. (A.4) by $\widetilde{\mathbf{L}}^{T}\left(k_{i}\right)$ for each reduced frequency, such that

$$
\begin{gathered}
\mathbf{C}^{(i j)} \tilde{\mathbf{L}}\left(k_{1}\right) \widetilde{\mathbf{L}}^{T}\left(k_{1}\right)=\overline{\mathbf{q}}_{i j}\left(k_{1}\right) \widetilde{\mathbf{L}}^{T}\left(k_{1}\right) \\
\mathbf{C}^{(i j)} \widetilde{\mathbf{L}}\left(k_{2}\right) \widetilde{\mathbf{L}}^{T}\left(k_{2}\right)=\overline{\mathbf{q}}_{i j}\left(k_{2}\right) \widetilde{\mathbf{L}}^{T}\left(k_{2}\right) \\
\vdots \\
\mathbf{C}^{(i j)} \widetilde{\mathbf{L}}\left(k_{3}\right) \widetilde{\mathbf{L}}^{T}\left(k_{3}\right)=\overline{\mathbf{q}}_{i j}\left(k_{3}\right) \widetilde{\mathbf{L}}^{T}\left(k_{3}\right)
\end{gathered}
$$


Thus, applying a summation of these equations, we have

$$
\mathbf{C}^{(i j)}\left\{\sum_{z=1}^{n_{k}}\left[\tilde{\mathbf{L}}\left(k_{z}\right) \tilde{\mathbf{L}}^{T}\left(k_{z}\right)\right]\right\}=\sum_{z=1}^{n_{k}}\left[\overline{\mathbf{q}}_{i j}\left(k_{z}\right) \tilde{\mathbf{L}}^{T}\left(k_{z}\right)\right]
$$

Finally, the coefficients of the vector $\mathbf{C}^{(i j)}$ can be obtained through the the following least-square solution:

$$
\mathbf{C}^{(i j)}=\left\{\sum_{z=1}^{n_{k}}\left[\overline{\mathbf{q}}_{i j}\left(k_{z}\right) \widetilde{\mathbf{L}}^{T}\left(k_{z}\right)\right]\right\}\left\{\sum_{z=1}^{n_{k}}\left[\widetilde{\mathbf{L}}\left(k_{z}\right) \tilde{\mathbf{L}}^{T}\left(k_{z}\right)\right]\right\}^{-1}
$$

Note that $\mathbf{C}^{(i j)}$ contain real coefficients not-dependent of the reduced frequency. Also, because the coefficients $\alpha_{x}$ are real numbers, we have

$$
\begin{aligned}
& q_{i j}(k)=C_{0}^{(i j)} \widetilde{L}_{0}+C_{1}^{(i j)} \widetilde{L}_{1}+\ldots+C_{r}^{(i j)} \widetilde{L}_{r} \\
& q_{i j}(k)=C_{0}^{(i j)}\left(\alpha_{0}^{(0)}\left(s^{\prime}\right)^{0}\right)+C_{1}^{(i j)}\left(\alpha_{0}^{(0)}\left(s^{\prime}\right)^{0}+\alpha_{1}^{(1)}\left(s^{\prime}\right)^{1}\right)+\ldots
\end{aligned}
$$

or, as an equivalent form,

$$
\begin{aligned}
q_{i j}(k)= & {\left[\sum_{z=0}^{r} C_{z}^{(i j)} \alpha_{0}^{(z)}\right]\left(s^{\prime}\right)^{0}+\left[\sum_{z=1}^{r} C_{z}^{(i j)} \alpha_{1}^{(z)}\right]\left(s^{\prime}\right)^{1}+\left[\sum_{z=2}^{r} C_{z}^{(i j)} \alpha_{2}^{(z)}\right]\left(s^{\prime}\right)^{2}+\left[\sum_{z=3}^{r} C_{z}^{(i j)} \alpha_{3}^{(z)}\right]\left(s^{\prime}\right)^{0}+\left[\sum_{z=4}^{r} C_{z}^{(i j)} \alpha_{4}^{(z)}\right]\left(s^{\prime}\right)^{1}+\left[\sum_{z=5}^{r} C_{z}^{(i j)} \alpha_{5}^{(z)}\right]\left(s^{\prime}\right)^{0} } \\
& +\left[\sum_{z=6}^{r} C_{z}^{(i j)} \alpha_{6}^{(z)}\right]\left(s^{\prime}\right)^{1}+\ldots
\end{aligned}
$$

In order to simplify the notation, Eq. (A.10) can be rewritten as

$$
q_{i j}(k)=\widetilde{q}_{i j}^{(0)}\left(s^{\prime}\right)^{0}+\widetilde{q}_{i j}^{(1)}\left(s^{\prime}\right)^{1}+\widetilde{q}_{i j}^{(2)}\left(s^{\prime}\right)^{2}+\widetilde{q}_{i j}^{(3)}\left(s^{\prime}\right)^{0}+\widetilde{q}_{i j}^{(4)}\left(s^{\prime}\right)^{1}+\widetilde{q}_{i j}^{(5)}\left(s^{\prime}\right)^{0}+\widetilde{q}_{i j}^{(6)}\left(s^{\prime}\right)^{1}+\ldots
$$

substituting $\left[s^{\prime}=(b / V) s\right]$ into Eq. (A.11) and grouping terms of the same order:

$$
q_{i j}(s)=\sum_{x} \tilde{q}_{i j}^{(x)}+\left(\frac{b}{V}\right)\left[\sum_{y} \tilde{q}_{i j}^{(y)}\right] s+\left(\frac{b}{V}\right)^{2}\left[\tilde{q}_{i j}^{(2)}\right] s^{2}
$$

where $x=[0,3,5,7, \ldots]$ and $y=[1,4,6,8, \ldots]$. Applying the inverse Laplace transform in the Eq. (A.12) and substituting its matrix form into right side of Eq. (1) $\left(q \mathbf{Q}(k) \mathbf{u}(t)=\mathbf{F}_{a}(t)\right)$, results

$$
\mathbf{F}_{a}(t)=q\left\{\sum_{x} \widetilde{\mathbf{Q}}^{(x)} \mathbf{u}(t)+\left(\frac{b}{V}\right) \sum_{y} \widetilde{\mathbf{Q}}^{(y)} \dot{\mathbf{u}}(t)+\left(\frac{b}{V}\right)^{2} \widetilde{\mathbf{Q}}^{(2)} \ddot{\mathbf{u}}(t)\right\}
$$

\section{References}

Albano, E., Rodden, W.P., 1969. A doublet-lattice method for calculating lift distributions on oscillating surfaces in subsonic flows. AIAA J. 7 (2), 279-285. Bueno, D.D., Gonçalves, P.J.P., Góes, L.C.S., 2014a. Aeroelastic stability analysis considering a continuous flight envelope. J. Fluids Struct. 49 , $716-727$.

Bueno, D.D., Sandoval Góes, L.C., Gonçalves, P.J.P., 2014b. Control of limit cycle oscillation in a three degrees of freedom airfoil section using fuzzy takagi-sugeno modeling. Shock Vib., 2014.

Bueno, D.D., Góes, L.C., Gonçalves, P.J., 2015. Flutter analysis including structural uncertainties. Meccanica, 1-9.

Bueno, D.D., Marqui, C.R., Sandoval Góes, L.C., Gonçalves, P.J.P., 2013. The use of gramian matrices for aeroelastic stability analysis. Math. Probl. Eng., 2013.

Dinu, A.D., Botez, R.M., Cotoi, I., 2005. Aerodynamic forces approximations using the chebyshev method for closed-loop aeroservoelasticity studies. Can. Aeronaut. Space J. 51 (4), 1-9.

Dowell, E.H., Hall, K.C., Thomas, J.P., Florea, R., Epureanu, B.I., Heeg, J., 1999. Reduced order models in unsteady aerodynamics. AIAA J..

Haase, D., Selmin, V., Winzell, B., 2003. Progress in Computational Flow-Structure Interaction - Results of the Project UNSI, supported by the European Union 1998 - 2000 Sci. Springer.

Haghighat, S., Martins, J.R.R.A., Liu, H.H.T., 2012. Aeroservoelastic design optimization of a flexible wing. J. Aircr. 49, 2.

Hesse, H., Palacios, R., 2007. Reduced-order aeroelastic models for dynamics of maneuvering flexible aircraft. AIAA J. 52 (8), $1717-1732$.

Iyanaga, S., Kawada, Y., 1980. Encyclopedic Dictionary of Mathematics. MIT Press.

Jones, R.T., 1940. The unsteady lift of a wing of finite aspect ratio. Tech. Rep. 681, National Advisory Committee for Aeronautics - NACA.

Karpel, M., 1982. Design for active flutter suppression and gust alleviation using state-space aeroelastic modeling. J. Aircr. 19 (3), $221-227$.

Karpel, M., 1990. Reduced-order aeroelastic models via dynamic residualization. J. Aircr. 27 (5), 449-455.

Karpel, M., 1981. Design for active and passive flutter suppression and gust alleviation. TR 3482, National Aeronautics and Space Administration - NASA.

Kim, T., Nagaraja, K.S., Bhatia, K.G., 2004. Order reduction of state-space aeroelastic models using optimal modal analysis. AIAA J. 41 (6), $1440-1448$.

Lieu, T., Farhat, C., 2007. Adaptation of aeroelastic reduced-order models and application to an f-16 configuration. AIAA J. 45 (6), $1244-1257$.

Munteanu, S., Rajadas, J., Nam, C., Chattopadhyay, A., 2005. Reduced-order-model approach for aeroelastic analysis involving aerodynamic and structural nonlinearities. AIAA J. 43 (3), 560-571.

Pahlavanloo, P., 2007. Dynamic aeroelastic simulation of the agard 445.6 wing using edge. Technical Report FOI-R-2259-SE, FOI - Swedish Defence Research Agency Defence and Security, System and Technology, SE-164 90 Stockholm. April.

Roger, K., 1977. Airplane math modelling methods for active control design. In: Structural Aspects of Active Control, In: Proceedings of the AGARD Conference, 
AGARD - Advisory Group for Aerospace Research \& Development, vol. 9 of 4, August, pp. 4.1-4.11.

Silva, W., Bartels, R., 2004. Development of reduced-order models for aeroelastic analysis and flutter prediction using the cfl3dv6.0 code. J. Fluids Struct. 19, $729-745$.

Silva, W., Vartio, E., Shimko, A., Kvaternik, R., Eure, K., Scott, R., 2006.Development of aeroservoelastic analytical models and gust load alleviationcontrol laws of a sensorcraft wind-tunnel model using measured data. In: Proceedings of the 47th AIAA/ASME/ASCE/AHS/ASC Structures, Structural Dynamics, and Materials Conference, Newport, Rhode Island, 1 - 4 May 2006

Thomas, J.P., Dowell, E.H., Hall, K.C., 2007. Using automatic differentiation to create a nonlinear reduced-order-model aerodynamic solver. AIAA J. 48 (1), 19-24.

Wright, J., Cooper, J., 2007. Introduction to Aircraft Aeroelasticity and Loads. AIAA education series. John Wiley \& Sons. URL 〈https://books.google.com.br/books? Id=gdEeAQAAIAAJ $\rangle$

Yates, E., 1988. Agard standard aeroelastic configurations for dynamic response i-wing 445.6. Tech. rep., Advisory Group for Aerospace Research and Development. Zwillinger, D., 1997. Handbook of Differential Equations, 3rd ed. Academic Press. 